\title{
Heat Transfer Characteristics of Pin-Fin Arrays with Ribs to Cool Combustor Liners*
}

\author{
Yutaka ODA**, Kenichiro TAKEISHI**, Yusuke MOTODA**, \\ Shinsuke SUGIMOTO** and Yoshiaki MIYAKE*** \\ ${ }^{* *}$ Osaka University, \\ 2-1, Yamadaoka, Suita, Osaka 565-0871, Japan \\ E-mail: takeishi@mech.eng.osaka-u.ac.jp

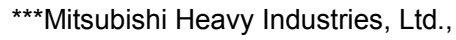

1200, O-aza Higashi Tanaka, Komaki, Aichi 485-8561, Japan

\begin{abstract}
Enhancement of heat transfer by pin-fin channels with turbulence promoters has been investigated with an aim to improve the cooling efficiency of combustor liners in gas turbines. Time-mean local Nusselt number profiles were obtained by naphthalene sublimation technique based on the heat/mass transfer analogy for Reynolds numbers ranging from 1000-10000. Two oblique rib-type turbulence promoters were tested and compared with no-rib cases. In addition, steady three-dimensional numerical simulations using a RANS model were performed. Installation of turbulence promoters is found to be effective in increasing the endwall heat transfer rate widely and uniformly. In addition, the ribs enhance heat transfer, especially in developing regions at the first and second pin-fin rows. Local high heat transfer regions were obtained in front of the ribs due to horseshoe vortices and downstream of the pins due to a longitudinal vortex.
\end{abstract}

Key words: Pin-Fins, Combustor, Jet Engine, Heat and Mass Transfer, Naphthalene Sublimation Method

\section{Introduction}

Convective heat transfer in confined channels with an array of circular pin fins has been investigated extensively in the past because of the importance of pin fins in a wide variety of engineering applications. Pin fins are commonly used for cooling the combustor liners in jet engines and gas turbines and the trailing edge regions of turbine blades and vanes. Since efficient cooling of combustor liners is important for a long life of high-temperature components, it is important to improve the cooling capability of pin-fin channels. Furthermore, from an environmental aspect, reducing the cooling airflow rate in combustors of industrial gas turbines leads to an increase of airflow rate available for pre-mixed type combustion, which is desirable for NOx reduction that requires plenty of air.

When pin fins are employed to cool combustor liners, turbine blades, and vanes, the space available for pin-fin arrays is limited. Hence, "short" pin fins, which have small height-to-diameter ratios on the order of unity, have been used in gas turbines. As reviewed by Chyu et al. ${ }^{(1)}$, the thermohydraulic performance of short pin-fin arrays has been widely investigated by Sparrow et al. ${ }^{(2)}$, Metzger et al. ${ }^{(3),(4)}$, VanFossen ${ }^{(5)}$, Chyu et al. ${ }^{(1)}$, and Chyu and Goldstein ${ }^{(6)}$. Armstrong and Winstanley ${ }^{(7)}$ summarized important results from the studies that were conducted in the 1980s in a review paper. Recently, Arik and Bunker ${ }^{(8)}$ reviewed pin-fin cooling in gas turbines. The parameters important in pin-fin cooling were identified as pin height-to-diameter ratio, lateral spacing, axial spacing, shape, in-line or 
staggered array pattern, and the number of pin-fin rows.

However, most research groups investigated basic and simple arrangements of pin-fin arrays with no additional devices to enhance heat transfer. Recently, Takeishi et al. ${ }^{(9)}$ investigated the overall heat transfer characteristics of pin fins with and without turbulence promoters (TP) on the endwall; they reported that TP enhance pin-fin heat transfer under actual engine conditions where fin efficiency becomes important because of the high heat transfer coefficient and low thermal conductivity of the superalloys used in the pin fins. The present study is an extension of an earlier study by Takeishi et al. ${ }^{(9)}$ to measure the local heat transfer coefficients, which are important in the cooling design of combustor liners and trailing edge sections of turbine vanes. The effect of TP on the endwall heat transfer in pin-fin channels was investigated in detail. This was done by measuring local Nusselt number profiles by the naphthalene sublimation method in mass transfer experiments. The naphthalene sublimation method is a sophisticated procedure in which a precision laser displacement sensor and an autotraverse system are used. In addition, to gain an insight into the heat transfer mechanism, numerical simulations were performed by using a low-Re $k-\varepsilon$ model for periodic fully developed flow and thermal fields in the corresponding pin-fin channels with ribs.

\section{Nomenclature}

$C_{p}$ : $\quad$ Specific heat of air at constant pressure

$D_{h}$ : Hydraulic diameter of a pin-fin channel

$d$ : Pin diameter

$e: \quad$ Height of turbulence promoter

$H: \quad$ Pin height

$h: \quad$ Heat transfer coefficient

$h_{d}: \quad$ Mass transfer coefficient

$\mathrm{n}: \quad$ Empirical constant

$P_{1}: \quad$ Spanwise pitch of pin fin

$P_{2}: \quad$ Streamwise pitch of pin fin

$p_{w}$ : $\quad$ Saturated naphthalene vapor pressure in air

$R: \quad$ Gas constant

Sc : $\quad$ Schmidt number

$t_{e}: \quad$ Flow exposure time

$T_{w}$ : Naphthalene surface temperature

$W: \quad$ Channel width

$\delta: \quad$ Sublimation depth of naphthalene layer

$\rho_{s}: \quad$ Density of solid naphthalene

\section{Experimental Method}

Figure 1 shows the experimental setup of a mass transfer measurement by the naphthalene sublimation method ${ }^{(10)}$. The test section is a rectangular channel equipped with pin-fin arrays. Both ends of the arrays are attached to the top and bottom walls of the channel. The channel is $280 \mathrm{~mm}$ long. Depending upon the test cases, which are described later, the height and width of the channel may be 17.5 and $20 \mathrm{~mm}$ or 18.7 and $35.6 \mathrm{~mm}$, respectively. Dry air is supplied from a compressor into the test section through a valve, rotameter, and pressure gauge. The entrance to the test section has a mesh screen followed by a contraction section to realize uniform flows upstream of the pin-fin arrays. As shown in Fig. 1, the downstream portion of the bottom surface of the channel can be separated, and a smooth naphthalene layer is molded over the surface by a casting method. 
In this study, four kinds of pin-fin channels with and without TP were tested. Figure 2 shows the basic arrangement of pin fins, in which they are placed in a staggered manner in terms of the mean flow direction. There were six rows of pin fins in the streamwise direction. There were either two or three pin fins in the spanwise direction; three pin fins comprise one perfect pin fin and two half pin fins. Important geometric parameters such as the pin diameter $d$, pin height $H$, aspect ratio $H / d$, spanwise pitch $P_{1}$, streamwise pitch $P_{2}$, and channel width $W$ are listed in Table 1 . From Table 1 , it is seen that Case 1 and Case 2 involved simple pin-fin channels with no TP and different $H / d$ to clarify the effect of $H / d$ on heat (mass) transfer. Case 3 and Case 4 involved pin-fin channels with TP. TP are oblique ribs placed between the pin fins and installed on the bottom surface of the channel, as shown in Fig. 2 for Case 3. The detailed geometric shapes and dimensions of the ribs are mentioned in Fig. 3 and Table 1. In this study, the rib in Case 4 is called an obliquely installed "rectangular rib," while that in Case 3 is called a "sheared rib" because it looks like it has been deformed by a shearing force. As listed in Table 1, the range of the Reynolds number in this experiment is from 1000-10000, which is based on the pin diameter and mean velocity at the cross section of the rectangular channel.

Each mass transfer experiment by naphthalene sublimation method was performed as follows. Firstly, reagent-grade (98\% pure) naphthalene crystals were melted in a pan with an induction heater until they boiled rapidly. After reaching a temperature of approximately $380 \mathrm{~K}$, the naphthalene was poured into a mold preheated at $330 \mathrm{~K}$. When the mold was cooled to room temperature, the naphthalene solidified; then, the cover plate of the mold was struck with a hammer to be removed. Secondly, the initial surface profile was scanned at regular intervals of 0.2 to $0.9 \mathrm{~mm}$ using a two-axis autotraverse equipment and a laser displacement sensor; the sensor had a measurement range of $\pm 1 \mathrm{~mm}$ with a resolution of 0.1 $\mu \mathrm{m}$ and linearity error of $0.3 \%$. Thirdly, after air blowing for 40 to $120 \mathrm{~min}$, the surface profile was scanned again. The duration of air blowing depended upon the Reynolds number. The local naphthalene sublimation depth was calculated as the local change of

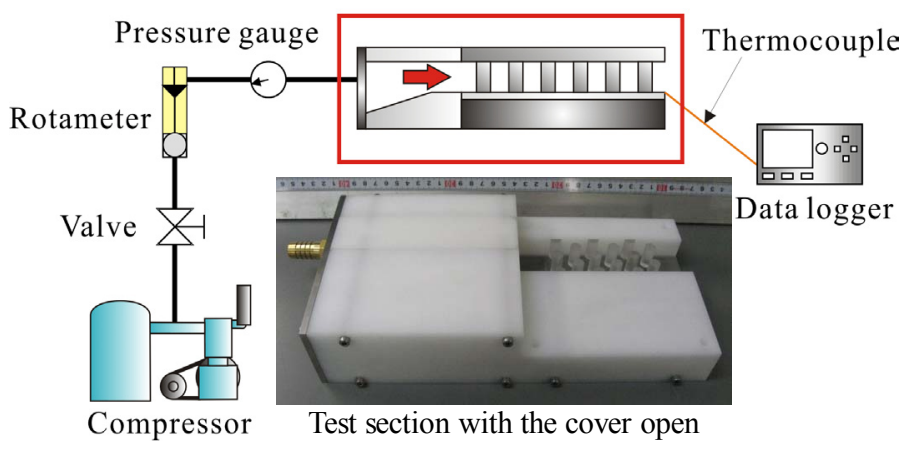

Fig. 1 Schematic of the experimental apparatus.
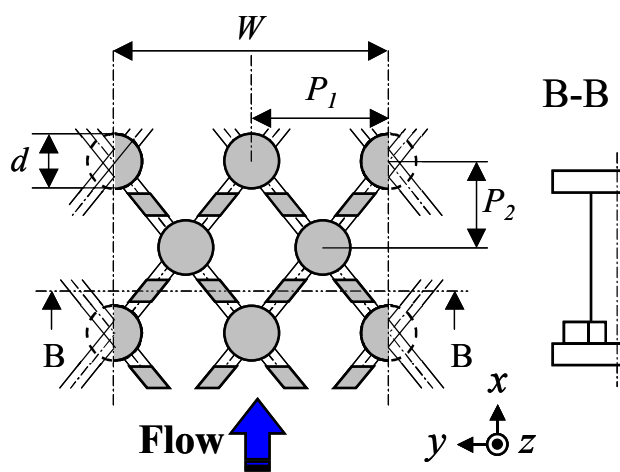

Fig. 2 Schematic of the pin-fin configuration in Case 3. 
Table. 1 Experimental conditions.

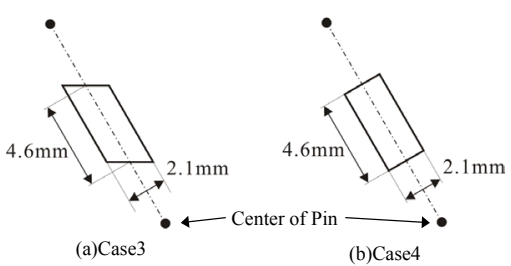

Fig. 3 Schematic of turbulence promoter (TP) configuration in Case 3 and Case 4.

\begin{tabular}{|c|c|c|c|c|}
\hline & & Case1 & Case2 & Case3,4 \\
\hline Diameter [mm] & $d$ & 5.0 & 9.0 & 9.0 \\
\hline Height [mm] & $H$ & 17.5 & 18.7 & 18.7 \\
\hline Aspect ratio & $H / d$ & 3.5 & 2.08 & 2.08 \\
\hline $\begin{array}{c}\text { Span wise pitch } \\
{[\mathrm{mm}]}\end{array}$ & $P_{l}$ & 10.0 & 17.8 & 17.8 \\
\hline $\begin{array}{c}\text { Stream wise } \\
\text { pitch [mm] }\end{array}$ & $P_{2}$ & 8.7 & 15.5 & 15.5 \\
\hline TP height [mm] & $e$ & - & - & 2.1 \\
\hline $\begin{array}{c}\text { Channel width } \\
{[\mathrm{mm}]}\end{array}$ & $W$ & 20.0 & 35.6 & 35.6 \\
\hline $\mathrm{Re}_{\mathrm{d}}$ & - & $10000,5000,2500,1000$ \\
\hline
\end{tabular}

surface position from the initial values.

The local mass transfer coefficient was calculated by using the following equation:

$$
h_{d}=\left(R T_{w} / p_{w}\right) \cdot\left(\rho_{s} \delta / t_{e}\right)
$$

Here, $R$ is the gas constant; $T_{w}$, the naphthalene surface temperature; $p_{w}$, the saturated vapor pressure of naphthalene in air; $\rho_{s}$, the density of solid naphthalene; $\delta$, the depth of naphthalene sublimation; and $t_{e}$, the flow exposure time. The thermophysical properties of naphthalene were obtained from the report published by Goldstein and Cho ${ }^{(10)}$. The local mass transfer coefficient can be converted to the local heat transfer coefficient by the analogy between heat and mass transfer.

$$
h=h_{d} \rho C_{p}(S c / P r)^{1-\mathrm{n}}
$$

Here, $\rho$ is the density of air; $C_{p}$, the specific heat at constant pressure; $S c$, the Schmidt number; $P r$, the Prandtl number; and $\mathrm{n}$, the empirical constant $(\mathrm{n}=0.4$ in this experiment).

\section{Numerical Method}

In this study, numerical simulations using FLUENT 6.3 were conducted to examine the heat transfer mechanism and compare the predicted results with the measured data obtained by naphthalene sublimation. $R e_{d}=5000$ was considered as the representative case for naphthalene sublimation. Based on the heat/mass transfer analogy, numerical analysis was performed for heat transfer instead of mass transfer.

In the present flow configurations, pin-fin geometries vary in a repeating manner along the mean flow direction, leading to a periodic fully developed flow after a sufficient entrance length. Since experimental results shown in the later sections indicate that the flow and heat transfer are fully developed after the fourth or fifth row, the present numerical analysis is intended to simulate periodic fully developed flow and heat transfer in the pin-fin channels. Figure 4 shows an example of the computational domain and mesh for Case 4. To achieve periodic flows with given mass flow rates, the local pressure gradient was solved: it was decomposed into the gradients of a periodic component and a linearly varying component. Iterations were needed until the given mass flow rate was achieved. In addition to the streamwise periodicity, the flow and heat transfer was assumed to be symmetric in the spanwise direction. Therefore, a symmetric boundary condition was applied to the spanwise boundaries of the domain shown in Fig. 4. Figure 5 shows top views of the meshes on the bottom surfaces in Case 3 and Case 4. Hexahedral meshes for 


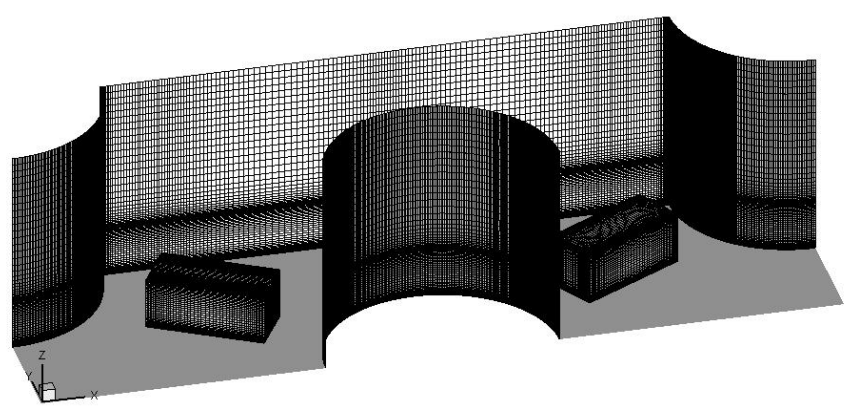

Fig. 4 Computational domain for Case 4.

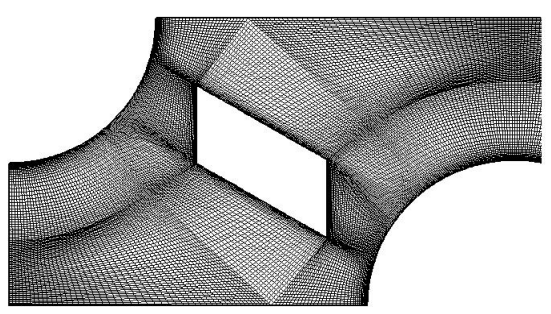

(a) Case 3

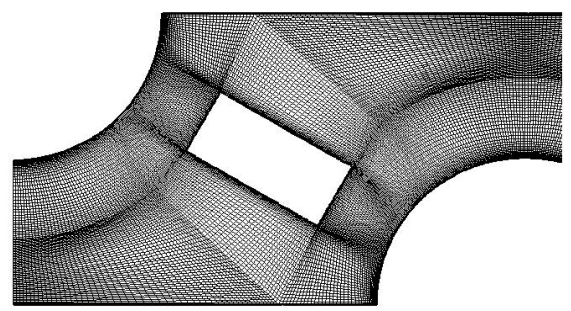

(b) Case 4

Fig. 5 Meshes on the bottom wall in (a) Case 3 and (b) Case 4.

the entire volume of the computational domain were formed by a multiblock method.

Owing to the limited computational resources available, the height of the computational domain is limited to $4 e$, while $H$ is approximately $9 e$ in experiments. Thus, a shear-free boundary condition is applied at the top boundary. For thermal boundary conditions, the naphthalene region, i.e., the uncovered endwall area on the bottom surface (shown as a gray area in Fig. 4) is considered to have a constant wall temperature of $350 \mathrm{~K}$. Since the surfaces of the pin fins and ribs were inactive for mass transfer in the experiment, adiabatic conditions were applied to simulate the experimental conditions; additional simulations were conducted under a constant temperature condition for all solid walls to examine the effect of rib installation on the overall heat transfer. To simulate periodic fully developed heat transfer, the inlet bulk mean temperature was fixed at $300 \mathrm{~K}$.

A low-Re $k$ - $\varepsilon$ model (AKN model) designed by Abe et al. ${ }^{(11)}$ was used as the turbulence model. In order to predict the turbulent heat fluxes, a constant turbulent Prandtl number $\operatorname{Pr}_{t}$ $=0.85$ was used by assuming a direct analogy between the turbulent heat transport and momentum transport. For stable computations, a first-order upwind differential scheme was used to discretize the convection terms for momentum, turbulent kinetic energy, and its dissipation rate. For the convective term of the energy equations, a second-order upwind scheme was employed. There were 747,000 cells in Case 2 and 2,920,000 each in Cases 3 and 4 . The grid spacing in the direction normal to the wall on the naphthalene surfaces satisfied $y^{+}<1$.

\section{Results and Discussion}

\subsection{Simple Pin-Fin Channels}

Figure 6 shows the contour map of the local time-mean Nusselt number obtained by naphthalene sublimation for Cases 1 and 2, which have different pin aspect ratios $H / d$ of 3.5 and 2.08, respectively, for $R e_{d}=5000$. Although the contour maps of the local Nusselt number for Cases 1 and 2 show similar profiles, the values of the local Nusselt numbers are higher in Case 2 than in Case 1. Local high Nusselt number regions are observed in front of each pin fin in both cases. This indicates that horseshoe vortices that promote heat transfer 


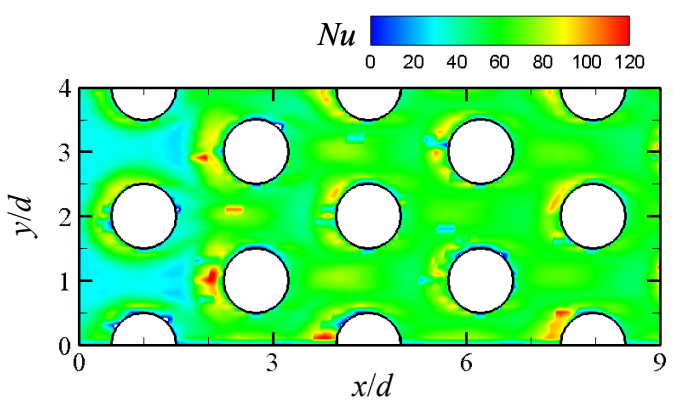

(a) Case 1

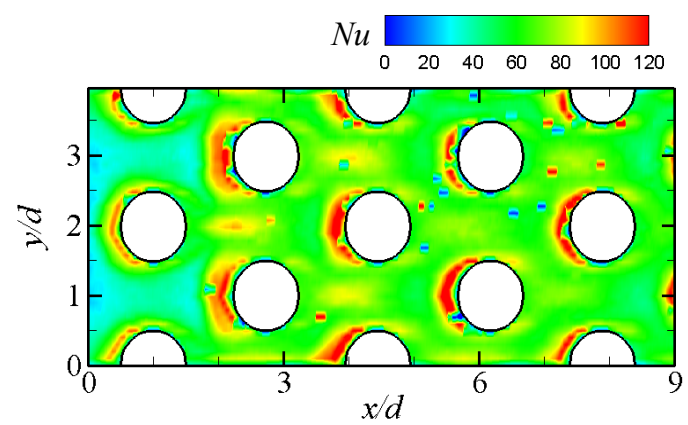

(b) Case 2

Fig. $6 N u$ distribution for $R e_{d}=5000$ for (a) Case 1 and (b) Case 2 .

are formed in these regions. After the second row, streaky patterns corresponding to high Nusselt numbers are also observed between the pin fins. This is because wakes from the upstream pins enhance heat transfer in the region between the pin fins.

Figure 7 shows the corresponding row-by-row averaged Nusselt number distribution for Cases 1 and 2. In the developing heat transfer region, both cases show a lower Nusselt number for the first row; the highest value corresponds to the second or third row. After the fourth row, the heat transfer seems to be fully developed. In addition, as expected from Fig. 6, the row-by-row averaged Nusselt number in Case 2 shows a higher Nusselt number than in Case 1, which had a larger $H / d$ than Case 2. This implies that the effective Reynolds number is larger in Case 2 because of a decrease in the effective cross-sectional area due to the displacement thickness of the momentum boundary layer being relatively larger when the pin aspect ratio is smaller. This can be confirmed by observing Fig. 8, which shows the relation between the averaged Nusselt number over the entire bottom surface and the Reynolds number $R e_{D h}$ based on the hydraulic diameter. The hydraulic diameter $D_{h}$ is four times the flow area divided by the wetted perimeter. As shown in Fig. 8, the Nusselt number in Cases 1 and 2 show similar trends when the horizontal axis represents $R e_{D h}$.
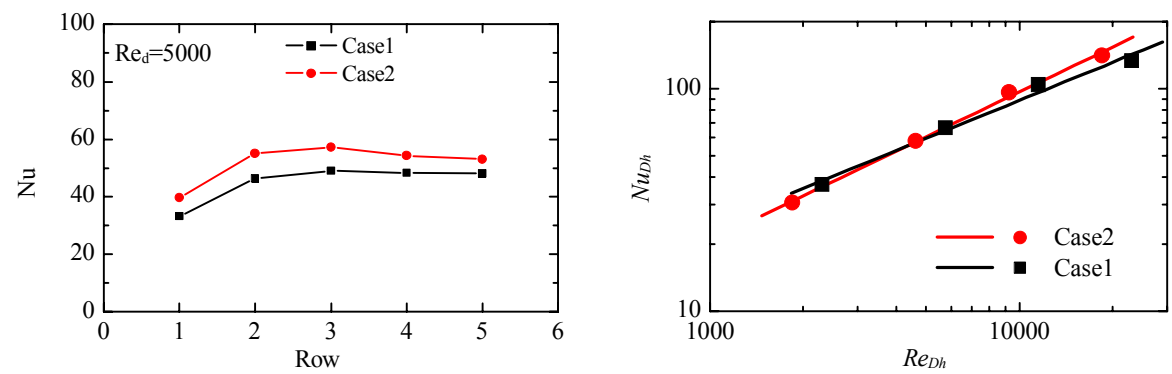

Fig. 7 Row-by-row averaged $N u$ for $R e_{d}=5000$. Fig. 8 Averaged $N u$ number vs. $R e_{D h}$. 


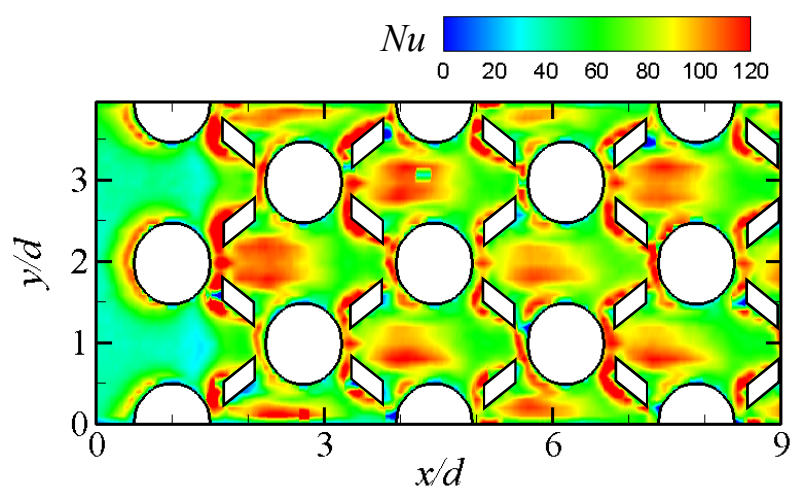

Fig. $9 \mathrm{Nu}$ distribution for $R e_{d}=5000$ for Case 3 .

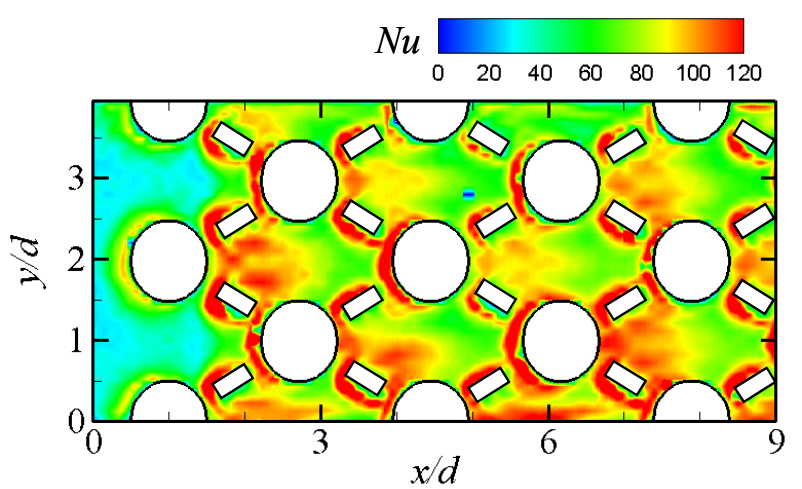

Fig. $10 N u$ distribution for $R e_{d}=5000$ for Case 4 .

\subsection{Pin-Fin Channels with Ribs}

Figure 9 shows the contour map of the local Nusselt number obtained by naphthalene sublimation for Case 3 at $R e_{d}=5000$. The endwall heat transfer is enhanced more widely and uniformly when sheared ribs are used. In particular, local high heat transfer regions are observed in front of the sheared ribs, indicating that horseshoe vortices that enhance heat transfer are formed in front of the sheared ribs. In addition, other high Nusselt number areas are observed downstream of the center of each pin. This indicates that flow separation at the rib-edge creates a strong secondary flow that impinges these regions.

Figure 10 shows the contour map of the Nusselt number for Case 4 at $R e_{d}=5000$. In terms of heat transfer enhancement, the contour map for Cases 3 and 4 are similar. In other words, the enhanced heat transfer regions spread more widely and uniformly than in the cases with the simple pin fins. Unlike in Case 3, the local high Nusselt number regions in front of each pin remain clear in Case 4, indicating that wakes from the rectangular ribs do not interfere considerably with the horseshoe vortices.

Figure 11 shows the corresponding row-by-row averaged Nusselt number profiles for

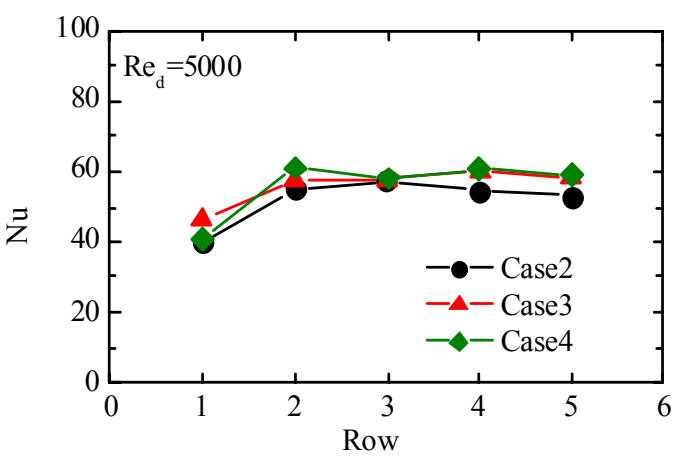

Fig. 11 Row-by-row averaged $N u$ at $R e_{d}=5000$ for Cases 3 and 4 . 


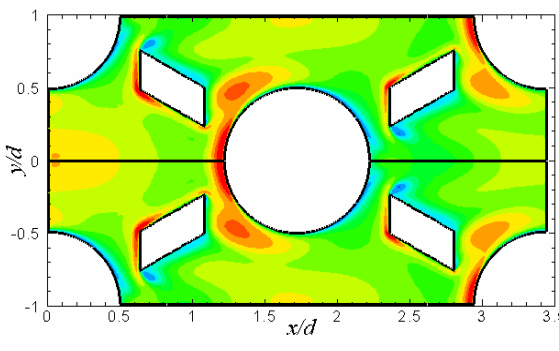

(a) Case 3

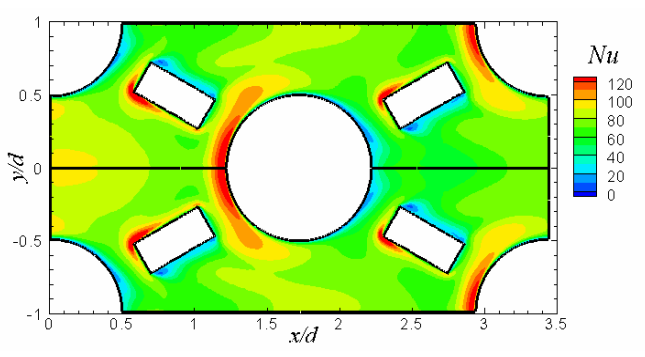

(b) Case 4

Fig. 12 Predicted local $N u$ at $R e_{d}=5000$ for (a) Case 3 and (b) Case 4.

Cases 2, 3, and 4. Installing ribs on the endwall of the pin fins is found to enhance heat transfer at the first or second rows, where heat transfer is developing. In addition, it also augments heat transfer at the fourth and fifth rows, where heat transfer seems to be fully developed. In realistic applications of pin fins to the cooling of jet engines and gas turbines, the available space and number of pins are limited. Hence, enhancement of the developing heat transfer regions of the pin-fins rows is an advantage of installation of ribs on the endwall.

\subsection{Numerical Analysis}

Figure 12 shows the numerically obtained local Nusselt number distribution for Cases 3 and 4 at $R e_{d}=5000$. A comparison with the experimental results, shown in Fig. 10, show that the following characteristics are predicted well by numerical simulations. There are local high Nusselt number regions in front of each pin due to the presence of horseshoe vortices; owing to these vortices, thermally fresh fluid from the main stream impinges the endwall. From the experimental observation for Case 3 (Fig. 9), the high $\mathrm{Nu}$ region in front of the pin fins are confirmed to be affected by the wake behind the sheared ribs. In addition, high Nusselt number areas at the edges of the rectangular ribs are also predicted for both Case 3 and Case 4. Furthermore, wake-induced streaky patterns with high Nusselt numbers are observed between the pin fins at $1.1<x / d<2.2$ and $0.7<y / d<1$. However, when compared with the experimental results, it is seen that the location of the heat transfer regions enhanced by flow reattachment is predicted to be far downstream, and the $N u$ values are underestimated. This discrepancy may be due to the difficulty in predicting such strong three-dimensional turbulent flows by linear eddy viscosity models. However, the present numerical analysis seems to predict the primary characteristics of flow and thermal fields, including the effect of secondary flows generated by ribs, in a reasonable manner to enable discussion of the heat transfer mechanism.

Figures 13 and 14 show the contour maps of the streamwise vorticity (top) and vertical velocity (bottom) for Cases 3 and 4 , respectively, at cross sections $x / P_{2}=0.25,0.5,0.75$, $1.0,1.25,1.5$, and 1.75. The Corresponding local Nusselt number distributions on the endwall are also shown in the figures. Note that the vertical velocity contours show only negative (downward) velocity with color. Positive (upward) vertical velocity is shown to be colorless in the velocity contour. As shown in both figures, the basic flow characteristics are similar to each other. Strong vorticity regions with negative and positive values are observed near the endwall at $x / P_{2}=0.75$ and 1.75 , respectively. These are due to longitudinal vortices generated by the edges of the rectangular ribs that reattach to the endwall. Negative vertical velocity is observed at $x / P_{2}=0.75$ and 1.75 , where longitudinal vortices exist. Thus, it is concluded that the longitudinal vortices enhance the endwall heat transfer and form streaky patterns of high Nusselt numbers in the regions. In addition, strong vertical flow impinging onto the endwall was observed along the pin-fin walls. This downward flow is a part of a horseshoe vortex that also greatly enhances the endwall heat transfer. 

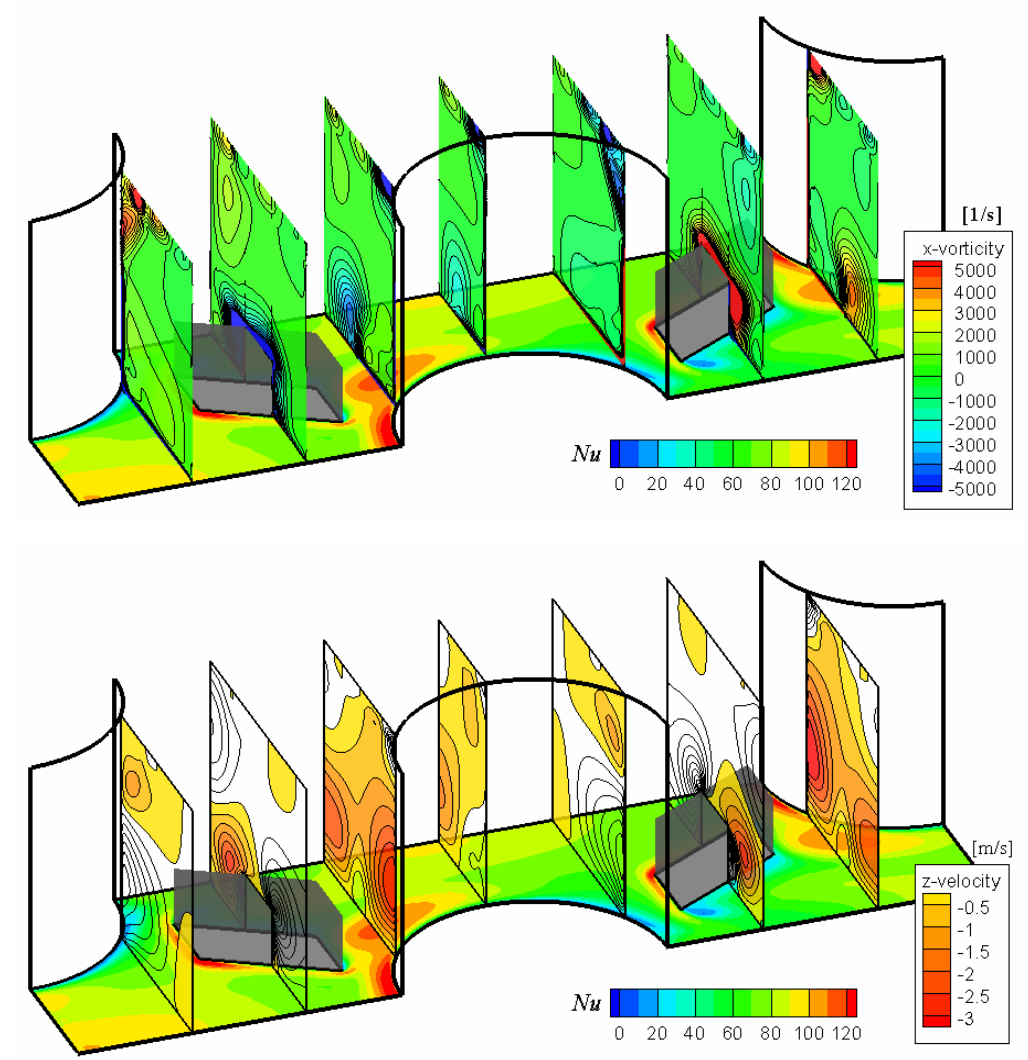

Fig. 13 Streamwise vorticity (top) and vertical velocity (bottom) at $R e_{d}=5000$ with local Nuseelt number on the endwall for Case 3.
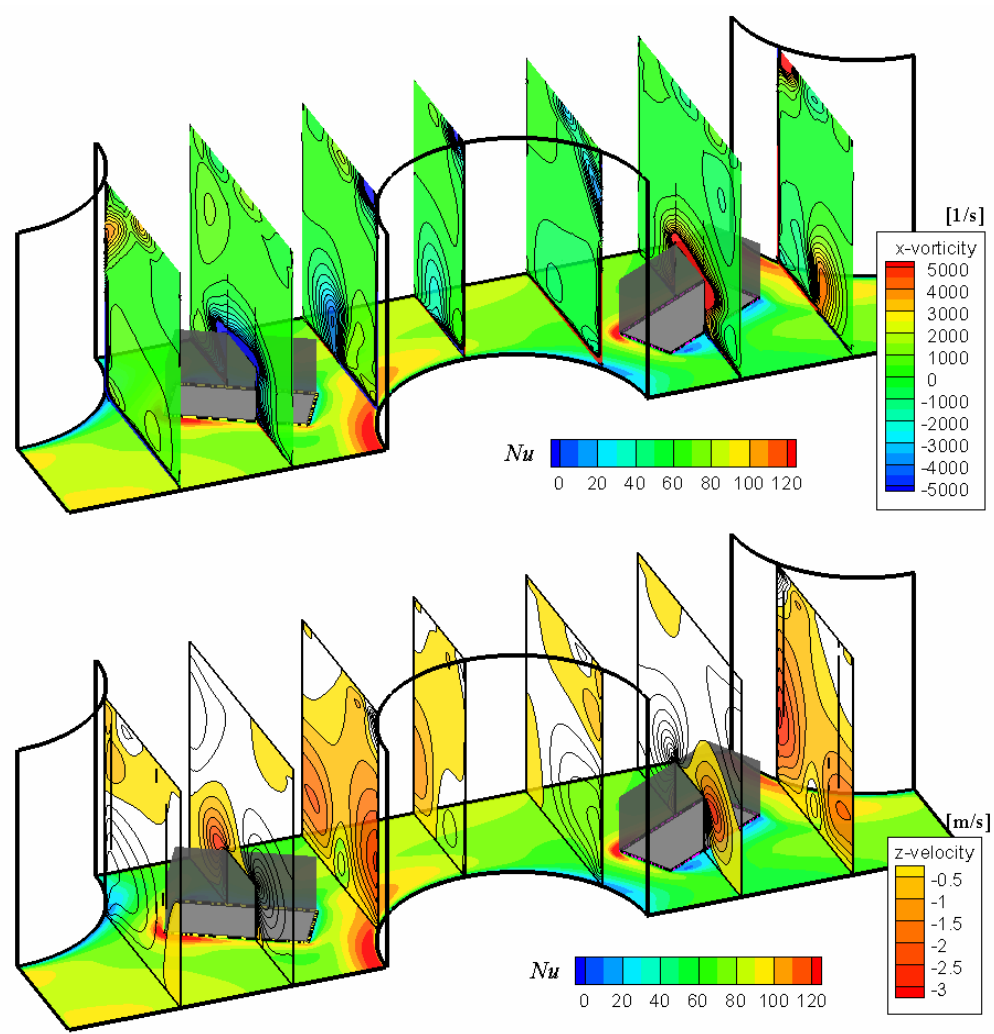

Fig. 14 Streamwise vorticity (top) and vertical velocity (bottom) at $R e_{d}=5000$ with local Nuseelt number on the endwall for Case 4. 
Table 2 Numerically predicted heat transfer characteristics of pin-fin channels with ribs

\begin{tabular}{lccc}
\hline & $\begin{array}{c}\text { Case 2 } \\
\text { (No rib) }\end{array}$ & $\begin{array}{c}\text { Case 3 } \\
\text { (Sheared rib) }\end{array}$ & $\begin{array}{c}\text { Case 4 } \\
\text { (Oblique rib) }\end{array}$ \\
\hline$\Delta p[\mathrm{~Pa}]$ & 134 & 162 & 151 \\
$N u_{m}:$ endwall & 73.8 & 67.9 & 66.3 \\
$N u_{m}:$ pin-fin & 106 & 102 & 102 \\
$N u_{m}:$ rib & - & 96.2 & 93.7 \\
$N u_{m}:$ total area & 90.7 & 88.2 & 87.4 \\
$A / A_{\text {norib }}$, area ratio & 1 & 1.13 & 1.13 \\
$N u_{m} \cdot\left(A / A_{\text {norib }}\right)$ & 90.7 & 99.7 & 98.8 \\
\hline
\end{tabular}

The preceding discussion on numerical results is based on simulations corresponding to mass transfer experiments, where only the endwall region (gray area in Fig. 4) is covered with a naphthalene layer. Thus, it is difficult to discuss the overall heat transfer characteristics of the present pin-fin channels with ribs. Therefore, additional simulations were conducted using constant temperature conditions for all of the solid walls in the computational domain. Table 2 lists the numerically predicted overall characteristics such as the pressure loss along the single unit length of $2 P_{2}$ and the area-averaged Nusselt number for each segment of endwall, pin fin, or rib of the pin-fin channels. The total area average of $N u$ and the area ratio, which is defined as the heat-transfer surface area normalized by that of the pin-fin channel with no rib, are also listed. In the last row, the product of the total $N u_{m}$ and area ratio, which expresses the effective $N u_{m}$ including the effect of the extended heat transfer area due to rib installations, are listed. As seen from items in the table, the overall heat transfer rate is enhanced by $10 \%$ in Case 3 (sheared rib); however, this was accompanied by a $21 \%$ increase in pressure loss. It is worth noting that the average $N u$ over the endwall area decreases when ribs are installed. This is because, as mentioned earlier, the heat transfer augmentation at the wake reattachment region is underestimated.

\section{Conclusions}

In this study, the effect of TP on the endwall heat transfer of pin-fin channels has been investigated experimentally and by numerical simulations for Reynolds numbers based on the pin diameter $R e_{d}=1000,2500,5000$, and 10000. Detailed distributions of the time-mean local Nusselt number on the endwall were obtained by naphthalene sublimation technique for pin-fin channels with and without TP, which were either oblique rectangular ribs or sheared ribs. Corresponding numerical simulations have also been conducted for fully developed regions where a periodic flow and heat transfer are assumed present. We obtained following conclusions.

(1) Endwall heat transfer is enhanced widely and uniformly by sheared or rectangular ribs. Local high heat transfer regions created by horseshoe vortices are observed in front of the sheared ribs; local high heat transfer regions are also observed downstream of the center of each pin due to a longitudinal vortex impinging the region.

(2) Installation of ribs on the endwall of the pin-fin channel enhances the heat transfer at the first or second row, where heat transfer is developing. This characteristic is advantageous in practical applications where the available space and number of pin fins are limited.

(3) In a simple pin-fin channel without ribs, the row-by-row averaged Nusselt number 
increases as the pin-height-to-diameter ratio decreases for the same Reynolds number based on pin diameter; this is because the effective Reynolds number increases due to a decrease in the effective cross-sectional area by the displacement thickness of the momentum boundary layer.

\section{Acknowledgements}

The authors thank the New Energy and Industrial Technology Development Organization (NEDO) for their financial support.

\section{References}

(1) Chyu, M.K., Hsing, Y.C., Shih, T.I.-P., Natarajan, V., Heat transfer contributions of pins and endwall in pin-fin arrays: Effect of thermal boundary condition modeling, Trans. ASME Journal of Turbomachinery, vol. 121, (1999), pp. 257-263.

(2) Sparrow, E.M., Ramsey, J.W., Altemani, C.A.C., Experiments on in-line pin fin arrays and performance comparisons with staggered arrays, Trans. ASME Journal of Heat Transfer, vol. 102, (1980), pp. 44-50.

(3) Metzger, D.E., Berry, R.A., Bronson, J.P., Developing heat transfer in rectangular ducts with staggered arrays of short pin fins, Trans. ASME Journal of Heat Transfer, vol. 104, (1982), pp. 700-706.

(4) Metzger, D.E., Fan, C.S., Haley, S.W., Effects of pin shape and array orientation on heat transfer and pressure loss in pin fin arrays, Trans. ASME Journal of Engineering for Gas Turbine and Power, vol. 106, (1984), pp. 252-257.

(5) VanFossen, G.J., Heat-transfer coefficients for staggered arrays of short pin fins, Trans. ASME Journal of Engineering for Power, vol. 104, (1982), pp. 268-274.

(6) Chyu, M.K., Goldstein, R.J., Influence of an array of wall-mounted cylinders on the mass transfer from a float surface, Int. J. Heat Mass Transfer, vol. 34, no. 9, (1991), pp. 2175-2186.

(7) Armstrong, J., Winstanley, D., A review of staggered array of pin fin heat transfer for tubine cooling applications, Trans. ASME Journal of Turbomachinery, vol. 110, (1988), pp. 94-103.

(8) Arik, M., Bunker, R.S., Electronics packaging cooling: Technologies from gas turbine engine cooling, Trans. ASME Journal of Electronic Packaging, vol. 128, (2006), pp. 215-225.

(9) Takeishi, K., Nakae, T., Watanabe, K., Hirayama, M., Heat transfer characteristics of a flow passage with long pin fins and improving heat transfer coefficient by adding turbulence promoters on a endwall, ASME Paper 2001-GT-178, (2001).

(10) Goldstein, R.J., Cho, H.H., A review of mass transfer measurements using naphthalene sublimation, Experimental Thermal and Fluid Sci., vol. 10, (1995), pp. 416-434.

(11) Abe, K., Kondoh, T., Nagano, Y., A new turbulence model for predicting fluid flow and heat transfer in separating and reattaching flows - I. Flow field calculations, Int. J. Heat Mass Transfer, vol. 37, (1994), pp. 139-151. 